\title{
Exponential Stability of Linear Discrete Systems with Variable Delays via Lyapunov Second Method
}

\author{
Josef Diblík \\ Central European Institute of Technology (CEITEC), Brno University of Technology, Brno, Czech Republic \\ Correspondence should be addressed to Josef Diblík; josef.diblik@ceitec.vutbr.cz
}

Received 12 July 2017; Accepted 24 October 2017; Published 23 November 2017

Academic Editor: Mustafa R. S. Kulenovic

Copyright (C) 2017 Josef Diblík. This is an open access article distributed under the Creative Commons Attribution License, which permits unrestricted use, distribution, and reproduction in any medium, provided the original work is properly cited.

\begin{abstract}
The paper investigates the exponential stability of a linear system of difference equations with variable delays $x(k+1)=A x(k)+$ $\sum_{i=1}^{s} B_{i}(k) x\left(k-m_{i}(k)\right), k=0,1, \ldots$, where $s \in \mathbb{N}, A$ is a constant square matrix, $B_{i}(k)$ are square matrices, $m_{i}(k) \in \mathbb{N} \cup\{0\}$, and $m_{i}(k) \leq m$ for an $m \in \mathbb{N}$. New criteria for exponential stability are derived using the method of Lyapunov functions and formulated in terms of the norms of matrices of linear terms and matrices solving an auxiliary Lyapunov equation. An exponential-type estimate of the norm of solutions is given as well. The efficiency of the derived criteria is numerically demonstrated by examples and their relations to the well-known results are discussed.
\end{abstract}

\section{Introduction}

Many mathematical real-life models are described by nonlinear systems of difference equations with delay. To derive local exponential stability results for such systems, it is reasonable to investigate the corresponding linear difference systems first. Such systems are the subject of the paper.

Recently, growing interest has been paid to investigating the stability of linear difference systems with delay (see, e.g., [1-11]).

The purpose of this paper is to give new sufficient conditions for the exponential stability of linear difference systems with variable delays

$$
\begin{aligned}
& x(k+1)=A x(k)+\sum_{i=1}^{s} B_{i}(k) x\left(k-m_{i}(k)\right), \\
& k=0,1, \ldots,
\end{aligned}
$$

where $A$ is an $n \times n$ constant matrix, $B_{i}(k)$ are $n \times n$ matrices, $m_{i}(k) \in \mathbb{N} \cup\{0\}, m_{i}(k) \leq m$ for an $m \in \mathbb{N}, s \in \mathbb{N}$, and $x=$ $\left(x_{1}, \ldots, x_{n}\right)^{T}:\{-m,-m+1, \ldots\} \rightarrow \mathbb{R}^{n}$. Note that the matrix of nondelayed terms in (1) is, in general, a time-dependent matrix due to the nonconstant perturbations of the matrix $A$ since the functions $m_{i}(k), i=1, \ldots, s$, can assume zero values for $k=0,1, \ldots$ The reason for selecting the constant matrix
$A$ in (1) is the subsequent use of the Lyapunov equation where only constant matrices are involved.

Simultaneously, we give an exponential estimate of the norms of solutions. The results are compared with the results published previously.

Recall the formulation of the Cauchy problem for (1). Given values $x_{k} \in \mathbb{R}, k=-m, \ldots, 0$, define a unique solution $x=x(k), k=-m,-m+1, \ldots$ of (1) by the conditions

$$
x(k)=x_{k}, \quad k=-m, \ldots, 0 .
$$

In this paper, for an arbitrary matrix $\mathscr{B}$, we use the norm $|\mathscr{B}|=\left(\lambda_{\max }\left(\mathscr{B}^{T} \mathscr{B}\right)\right)^{1 / 2}$, which, for vectors $x=\left(x_{1}, \ldots, x_{n}\right)^{T}$, implies the norm $|x|=\left(\sum_{i=1}^{n} x_{i}^{2}\right)^{1 / 2}$. Moreover, denote by $\lambda_{\text {max }}(\mathscr{A})$ and $\lambda_{\min }(\mathscr{A})$ the maximum and minimum eigenvalues of a positive definite symmetric matrix $\mathscr{A}$, respectively. Also, we define $\varphi(\mathscr{A}):=\lambda_{\max }(\mathscr{A}) / \lambda_{\min }(\mathscr{A})$.

Recall the basic definition related to stability (we refer, e.g., to [12]).

Definition 1. A trivial solution $x(k)=0, k=-m,-m+1, \ldots$, of (1) is called Lyapunov stable if, for a given $\varepsilon>0$, there exists $\delta(\varepsilon)>0$ such that, for any other solution $x(k)$, one has $|x(k)|<\varepsilon$ for $k=0,1, \ldots$, provided that $\|x(0)\|_{m}<\delta(\varepsilon)$, where

$$
\|x(0)\|_{m}:=\max \{|x(i)|, i=-m,-m+1, \ldots, 0\} .
$$


If, in addition, $\lim _{k \rightarrow+\infty}|x(k)|=0$, the trivial solution is called asymptotic stable. The trivial solution of system (1) is called Lyapunov exponentially stable if there exist constants $N>0$ and $\theta \in(0,1)$ such that $|x(k)| \leq N\|x(0)\|_{m} \theta^{k}, k=1,2, \ldots$

Note that Lyapunov exponential stability of the trivial solution of system (1) is equivalent with global exponential stability, defined, for example, in [9, Definition 2.1] if linear systems are considered.

It is obvious that system (1) can be transformed into a system with constant delays

$$
\begin{array}{r}
x(k+1)=\left(A+B_{0}^{*}(k)\right) x(k)+\sum_{i=1}^{m} B_{i}^{*}(k) x\left(k-m_{i}^{*}\right), \\
k=0,1, \ldots,
\end{array}
$$

where $B_{i}^{*}(k), i=0, \ldots, m$, are suitable $n \times n$ matrices and $m_{i}^{*}$ are natural numbers such that $m_{1}^{*}=1<m_{2}^{*}=2<\cdots<$ $m_{m}^{*}=m$. Initial problem (2) defines the same solution to system (4) as for system (1).

It should, however, be emphasized that such a transformation does not simplify the problem itself because the matrices $B_{i}^{*}(k), i=1, \ldots, m$, remain variable and, for example, the method based on the study of the corresponding characteristic equation is not applicable.

The paper studies the exponential stability of (1) by the second Lyapunov method. Recall the well-known fact, necessary for this method to be applied, that when the stability of linear discrete equations is investigated by Lyapunov function $V(x)=x^{T} H x$ with an $n \times n$ positive definite symmetric matrix $H$, an important role is played by what is called the Lyapunov equation

$$
A^{T} H A-H=-C
$$

where $A$ is a given $n \times n$ matrix and $C$ is an $n \times n$ matrix. The linear system $x(k+1)=A x(k), k=0,1, \ldots$, is exponentially stable (i.e., $\rho(A)<1$, where $\rho$ is the spectral radius of $A$ defined by $\rho(A)=\max \{|\lambda|: \lambda \in \sigma(A)\}, \sigma(A):=\{z \in$ $\mathbb{C}: \operatorname{det}(A-z E)=0\}$ is the set of all eigenvalues of $A$, and $E$ is the unit matrix), if and only if, for an arbitrary positive definite symmetric $n \times n$ matrix $C$, the matrix equation (5) has a unique solution-a positive definite symmetric matrix $H([12,13])$.

The remaining part of the paper is organized as follows. In Section 2, exponential stability of system (1) and exponential estimates are investigated. The case of constant matrices is treated in Section 3. Section 4 contains a concluding discussion, comparisons, and examples demonstrating the results obtained and their independence of the well-known results.

\section{Exponential Stability}

Sufficient conditions for the exponential stability of (1) and an estimate of solutions are given by the following theorem. A natural condition (tacitly assumed in the paper) is that $|A|+$ $\sum_{i=1}^{s}\left|B_{i}(k)\right|>0$ for any $k=0,1, \ldots$ In the proof, we utilize a
Lyapunov function of the form $V(x)=x^{T} H x$. Obviously, we have

$$
\lambda_{\min }(H)|x|^{2} \leq V(x) \leq \lambda_{\max }(H)|x|^{2} .
$$

Finally, define auxiliary numbers

$$
\begin{aligned}
& L(k, H)=\lambda_{\max }(H)-\lambda_{\min }(C)+\sum_{j=1}^{s}\left|A^{T} H B_{j}(k)\right|, \\
& k=0,1, \ldots, \\
& L_{i}(k, H) \\
& \quad=\lambda_{\min }(H) \\
& \quad-\varphi(H)\left[\left|A^{T} H B_{i}(k)\right|+\sum_{j=1}^{s}\left|B_{i}^{T}(k) H B_{j}(k)\right|\right], \\
& \quad i=1, \ldots, s, k=0,1, \ldots
\end{aligned}
$$

Theorem 2. Let $\rho(A)<1, C$ be a fixed positive definite matrix, let matrix $H$ solve the corresponding Lyapunov matrix equation (5)

$$
L(k, H)>0, \quad k=0,1, \ldots,
$$

and let a number $q \in(0,1)$ exist such that

$$
\begin{array}{r}
L(k, H)-\sum_{i=1}^{s} L_{i}(k, H) \leq q \lambda_{\max }(H)-s \lambda_{\text {min }}(H), \\
k=0,1, \ldots .
\end{array}
$$

Then, the system with delay (1) is exponentially stable and, for an arbitrary solution $x=x(k)$, the estimate

$$
|x(k)| \leq \sqrt{\varphi(H)}\|x(0)\|_{m} q^{k / 2(m+1)}, \quad k \geq 0,
$$

holds.

Proof. Set

$$
\begin{array}{r}
\Theta(k, H) \\
:=\frac{1}{\lambda_{\max }(H)}\left[L(k, H)-\sum_{i=1}^{s} L_{i}(k, H)+s \lambda_{\min }(H)\right], \\
k=0,1, \ldots .
\end{array}
$$

First we show that

$$
0<\Theta(k, H) \leq q, \quad k=0,1, \ldots
$$


The right-hand side inequality in (12) is an obvious consequence of (8) and (9). Let us consider the expression in the square brackets in (11). We get

$$
\begin{aligned}
& L(k, H)-\sum_{i=1}^{s} L_{i}(k, H)+s \lambda_{\min }(H)=L(k, H) \\
& -\sum_{i=1}^{s}\left[\lambda_{\min }(H)\right. \\
& \left.-\varphi(H)\left(\left|A^{T} H B_{i}(k)\right|+\sum_{j=1}^{s}\left|B_{i}^{T}(k) H B_{j}(k)\right|\right)\right] \\
& +s \lambda_{\min }(H)=L(k, H)+\varphi(H) \sum_{i=1}^{s}\left(\left|A^{T} H B_{i}(k)\right|\right. \\
& \left.+\sum_{j=1}^{s}\left|B_{i}^{T}(k) H B_{j}(k)\right|\right)>\varphi(H) \sum_{i=1}^{s}\left(\left|A^{T} H B_{i}(k)\right|\right. \\
& \left.+\sum_{j=1}^{s}\left|B_{i}^{T}(k) H B_{j}(k)\right|\right) \geq 0,
\end{aligned}
$$

and $\Theta(k, H)>0, k=0,1, \ldots$ Inequality (12) is verified.

Now we prove that an exponential rate of convergence (10) is valid.

Calculating the first difference of the Lyapunov function $V(x(k))$, where $x=x(k), k \geq-m,-m+1, \ldots$, is a solution of the system with delay (1), for $k \geq 0$, we get

$$
\begin{aligned}
& \Delta V(x(k))=V(x(k+1))-V(x(k))=x^{T}(k+1) \\
& \cdot H x(k+1)-x^{T}(k) H x(k)=[A x(k) \\
& \left.+\sum_{i=1}^{s} B_{i}(k) x\left(k-m_{i}(k)\right)\right]^{T} H[A x(k) \\
& \left.+\sum_{i=1}^{s} B_{i}(k) x\left(k-m_{i}(k)\right)\right]-x^{T}(k) H x(k) \\
& =-x^{T}(k)\left[H-A^{T} H A\right] x(k)+2 \sum_{i=1}^{s} x^{T}(k) \\
& \cdot A^{T} H B_{i}(k) x\left(k-m_{i}(k)\right)+\sum_{i=1}^{s} x^{T}\left(k-m_{i}(k)\right) \\
& \cdot B_{i}^{T}(k) H B_{i}(k) x\left(k-m_{i}(k)\right) \\
& +\sum_{i, j=1, i \neq j}^{s} x^{T}\left(k-m_{i}(k)\right) B_{i}^{T}(k) H B_{j}(k) \\
& \cdot x\left(k-m_{j}(k)\right) .
\end{aligned}
$$

Applying the Lyapunov matrix equation (5), we obtain

$$
\begin{aligned}
& \Delta V(x(k)) \leq-\lambda_{\min }(C)|x(k)|^{2}+2 \sum_{i=1}^{s}\left|A^{T} H B_{i}(k)\right| \\
& \cdot|x(k)|\left|x\left(k-m_{i}(k)\right)\right|+\sum_{i=1}^{s}\left|B_{i}^{T}(k) H B_{i}(k)\right| \\
& \cdot\left|x\left(k-m_{i}(k)\right)\right|^{2}+2 \sum_{i, j=1, i<j}^{s}\left|B_{i}^{T}(k) H B_{j}(k)\right| \\
& \cdot\left|x^{T}\left(k-m_{i}(k)\right)\right|\left|x\left(k-m_{j}(k)\right)\right| \text {, } \\
& \Delta V(x(k)) \leq-\lambda_{\min }(C)|x(k)|^{2}+\sum_{i=1}^{s}\left|A^{T} H B_{i}(k)\right| \\
& \cdot\left[|x(k)|^{2}+\left|x\left(k-m_{i}(k)\right)\right|^{2}\right]+\sum_{i=1}^{s}\left|B_{i}^{T}(k) H B_{i}(k)\right| \\
& \cdot\left|x\left(k-m_{i}(k)\right)\right|^{2}+\sum_{i, j=1, i<j}^{s}\left|B_{i}^{T}(k) H B_{j}(k)\right| \\
& \cdot\left[\left|x\left(k-m_{i}(k)\right)\right|^{2}+\left|x\left(k-m_{j}(k)\right)\right|^{2}\right] \\
& =\left[-\lambda_{\min }(C)+\sum_{i=1}^{s}\left|A^{T} H B_{i}(k)\right|\right]|x(k)|^{2} \\
& +\sum_{i=1}^{s}\left[\left|A^{T} H B_{i}(k)\right|+\left|B_{i}^{T}(k) H B_{i}(k)\right|\right] \\
& \cdot\left|x\left(k-m_{i}(k)\right)\right|^{2}+\sum_{i, j=1, i<j}^{s}\left|B_{i}^{T}(k) H B_{j}(k)\right| \\
& \cdot\left[\left|x\left(k-m_{i}(k)\right)\right|^{2}+\left|x\left(k-m_{j}(k)\right)\right|^{2}\right] \text {. }
\end{aligned}
$$

From inequalities (6), we immediately get

$$
\begin{aligned}
-|x(k)|^{2} & \leq-\frac{V(x(k))}{\lambda_{\max }(H)}, \\
\left|x\left(k-m_{i}(k)\right)\right|^{2} & \leq \frac{V\left(x\left(k-m_{i}(k)\right)\right)}{\lambda_{\text {min }}(H)}, \quad i=1, \ldots, s
\end{aligned}
$$

and, using (9),

$$
\begin{array}{r}
-\lambda_{\min }(C)+\sum_{i=1}^{s}\left|A^{T} H B_{i}(k)\right|=L(k, H)-\lambda_{\max }(H) \\
<L(k, H)-q \lambda_{\max }(H) \leq \sum_{i=1}^{s} L_{i}(k, H)-s \lambda_{\min }(H) \\
=-\varphi(H) \sum_{i=1}^{s}\left[\left|A^{T} H B_{i}(k)\right|+\left|B_{i}^{T}(k) H B_{i}(k)\right|\right] \leq 0,
\end{array}
$$


that is,

$$
\begin{aligned}
& {\left[-\lambda_{\min }(C)+\sum_{i=1}^{s}\left|A^{T} H B_{i}(k)\right|\right]|x(k)|^{2}} \\
& \leq\left[-\lambda_{\min }(C)+\sum_{i=1}^{s}\left|A^{T} H B_{i}(k)\right|\right] \frac{V(x(k))}{\lambda_{\max }(H)} .
\end{aligned}
$$

Now, (15) implies

$$
\begin{aligned}
& V(x(k+1)) \leq \frac{1}{\lambda_{\max }(H)}\left[\lambda_{\max }(H)-\lambda_{\min }(C)\right. \\
& \left.+\sum_{i=1}^{s}\left|A^{T} H B_{i}(k)\right|\right] V(x(k))+\frac{1}{\lambda_{\min }(H)} \\
& \cdot \sum_{i=1}^{s}\left[\left|A^{T} H B_{i}(k)\right|+\left|B_{i}^{T}(k) H B_{i}(k)\right|\right] \\
& \cdot V\left(x\left(k-m_{i}(k)\right)\right)+\frac{1}{\lambda_{\min }(H)} \\
& \cdot \sum_{i, j=1, i<j}^{s}\left|B_{i}^{T}(k) H B_{j}(k)\right|\left[V\left(x\left(k-m_{i}(k)\right)\right)\right. \\
& \left.+V\left(x\left(k-m_{j}(k)\right)\right)\right] \\
& =\left[\text { due to property }\left|B_{i}^{T}(k) H B_{j}(k)\right|\right. \\
& \left.=\left|B_{j}^{T}(k) H B_{i}(k)\right|\right]=\frac{1}{\lambda_{\max }(H)}\left[\lambda_{\max }(H)\right. \\
& \left.-\lambda_{\min }(C)+\sum_{i=1}^{s}\left|A^{T} H B_{i}(k)\right|\right] V(x(k)) \\
& +\frac{1}{\lambda_{\min }(H)}\left[\left|A^{T} H B_{1}(k)\right|+\sum_{j=1}^{s}\left|B_{1}^{T}(k) H B_{j}(k)\right|\right] \\
& \cdot V\left(x\left(k-m_{1}(k)\right)\right)+\cdots \\
& +\frac{1}{\lambda_{\min }(H)}\left[\left|A^{T} H B_{s}(k)\right|+\sum_{j=1}^{s}\left|B_{s}^{T}(k) H B_{j}(k)\right|\right] \\
& \cdot V\left(x\left(k-m_{s}(k)\right)\right) \text {, }
\end{aligned}
$$

or

$$
\begin{aligned}
V(x(k+1)) \leq & \frac{L(k, H)}{\lambda_{\max }(H)} V(x(k)) \\
& +\frac{1}{\lambda_{\max }(H)}\left[\lambda_{\min }(H)-L_{1}(k, H)\right] \\
& \cdot V\left(x\left(k-m_{1}(k)\right)\right)+\cdots \\
+ & \frac{1}{\lambda_{\max }(H)}\left[\lambda_{\min }(H)-L_{s}(k, H)\right] \\
& \cdot V\left(x\left(k-m_{s}(k)\right)\right) .
\end{aligned}
$$

Now we use inequality (20) to derive an exponential estimate of (10). Define

$$
v_{0}:=\max \{V(x(0)), V(x(-1)), \ldots, V(x(-m))\}
$$

By (6), it is easy to see that

$$
\begin{aligned}
v_{0} & \leq \lambda_{\max }(H) \max \{|x(0)|,|x(-1)|, \ldots,|x(-m)|\} \\
& =\lambda_{\max }(H)\|x(0)\|_{m}^{2} .
\end{aligned}
$$

Set $k=0$. Then, (20) yields

$$
\begin{aligned}
& V(x(1)) \leq \frac{L(0, H)}{\lambda_{\max }(H)} V(x(0))+\frac{1}{\lambda_{\max }(H)}\left[\lambda_{\min }(H)\right. \\
& \left.-L_{1}(0, H)\right] V\left(x\left(-m_{1}(0)\right)\right)+\cdots \\
& \quad+\frac{1}{\lambda_{\max }(H)}\left[\lambda_{\min }(H)-L_{s}(0, H)\right] \\
& \cdot V\left(x\left(-m_{s}(0)\right)\right) \leq \frac{1}{\lambda_{\max }(H)}[L(0, H) \\
& \left.-L_{1}(0, H)-\cdots-L_{s}(0, H)+s \lambda_{\min }(H)\right] v_{0} \\
& =\Theta(0, H) v_{0} \leq q v_{0} \leq q^{1 /(m+1)} v_{0} .
\end{aligned}
$$

By induction, we can prove

$$
V(x(k)) \leq q^{k /(m+1)} v_{0}, \quad k \geq-m .
$$

For $k=-m,-m+1, \ldots, 0$, inequality (24) holds since, by (21), $V(x(k)) \leq v_{0} \leq q^{k /(m+1)} v_{0}$. Inequality (24) holds for $k=1$ as well since, by (23), $V(x(1)) \leq q^{1 /(m+1)} v_{0}$. Let (24) be valid for every $k=-m,-m+1, \ldots, k^{*}$, where $k^{*}>1$. We prove that it holds for $k^{*}+1$ as well. From (20) we get

$$
\begin{aligned}
& V\left(x\left(k^{*}+1\right)\right) \leq \frac{L\left(k^{*}, H\right)}{\lambda_{\max }(H)} V\left(x\left(k^{*}\right)\right) \\
& +\frac{1}{\lambda_{\max }(H)}\left[\lambda_{\min }(H)-L_{1}\left(k^{*}, H\right)\right] \\
& \cdot V\left(x\left(k^{*}-m_{1}\left(k^{*}\right)\right)\right)+\cdots \\
& +\frac{1}{\lambda_{\max }(H)}\left[\lambda_{\min }(H)-L_{s}\left(k^{*}, H\right)\right] \\
& \cdot V\left(x\left(k^{*}-m_{s}\left(k^{*}\right)\right)\right) \leq \frac{L\left(k^{*}, H\right)}{\lambda_{\max }(H)} q^{k^{*} /(m+1)} v_{0} \\
& +\frac{1}{\lambda_{\max }(H)}\left[\lambda_{\min }(H)-L_{1}\left(k^{*}, H\right)\right] \\
& \cdot q^{\left(k^{*}-m_{1}\left(k^{*}\right)\right) /(m+1)} v_{0}+\cdots+\frac{1}{\lambda_{\max }(H)}\left[\lambda_{\min }(H)\right.
\end{aligned}
$$




$$
\begin{aligned}
& \left.-L_{s}\left(k^{*}, H\right)\right] q^{\left(k^{*}-m_{s}\left(k^{*}\right)\right) /(m+1)} v_{0}=\left[\frac{L\left(k^{*}, H\right)}{\lambda_{\max }(H)}\right. \\
& \cdot q^{m /(m+1)}+\frac{1}{\lambda_{\max }(H)}\left[\lambda_{\min }(H)-L_{1}\left(k^{*}, H\right)\right] \\
& \cdot q^{\left(m-m_{1}\left(k^{*}\right)\right) /(m+1)}+\cdots \\
& +\frac{1}{\lambda_{\max }(H)}\left[\lambda_{\min }(H)-L_{s}\left(k^{*}, H\right)\right] \\
& \left.\cdot q^{\left(m-m_{s}\left(k^{*}\right)\right) /(m+1)}\right] q^{\left(k^{*}-m\right) /(m+1)} v_{0} \leq\left[\frac{L\left(k^{*}, H\right)}{\lambda_{\max }(H)}\right. \\
& +\frac{1}{\lambda_{\max }(H)}\left[\lambda_{\min }(H)-L_{1}\left(k^{*}, H\right)\right]+\cdots \\
& +\frac{1}{\lambda_{\max }(H)}\left[\lambda_{\min }(H)-L_{s}\left(k^{*}, H\right)\right] \\
& \cdot q^{\left(k^{*}-m\right) /(m+1)} v_{0}=\Theta\left(k^{*}, H\right) \cdot q^{\left(k^{*}-m\right) /(m+1)} v_{0} \leq q \\
& \cdot q^{\left(k^{*}-m\right) /(m+1)} v_{0}=q^{\left(k^{*}+1\right) /(m+1)} v_{0} .
\end{aligned}
$$

So, (24) holds for every $k \geq-m$. Utilizing (6), (22), and (24), we obtain

$$
\begin{aligned}
& \lambda_{\min }(H)|x(k)|^{2} \leq V(x(k)) \leq q^{k /(m+1)} v_{0} \\
& \leq q^{k /(m+1)} \lambda_{\max }(H)\|x(0)\|_{m}^{2}, \\
& k \geq 0 \text {, }
\end{aligned}
$$

or

$$
\begin{aligned}
|x(k)| & \leq \sqrt{\frac{\lambda_{\max }(H)}{\lambda_{\min }(H)}} q^{k / 2(m+1)}\|x(0)\|_{m} \\
& =\sqrt{\varphi(H)}\|x(0)\|_{m} q^{k / 2(m+1)}, \quad k \geq 0 ;
\end{aligned}
$$

that is, the convergence of solutions can be described by the exponential law (10).

\section{Exponential Stability in the Case of Constant Matrices}

The result of Theorem 2 can be improved if matrices $B_{i}(k)$, $i=1, \ldots, s$, in (1) are constant; that is, $B_{i}(k) \equiv B_{i}=$ const. In such a case, the numbers $L(k, H), L_{i}(k, H), i=1, \ldots, s$, do not depend on $k$ and are constant as well. We redefine them as follows:

$$
\begin{aligned}
L(H) & :=\lambda_{\max }(H)-\lambda_{\min }(C)+\sum_{j=1}^{s}\left|A^{T} H B_{j}\right|, \\
L_{i}(H) & :=\lambda_{\min }(H)
\end{aligned}
$$

$$
\begin{aligned}
&-\varphi(H)\left[\left|A^{T} H B_{i}\right|+\sum_{j=1}^{s}\left|B_{i}^{T} H B_{j}\right|\right] \\
& i=1, \ldots, s .
\end{aligned}
$$

In such a case, the function $\Theta(k, H), k \geq 0$, defined by (11), does not depend on $k$ either and, in the following, we use a constant

$$
\begin{aligned}
& \Theta(H) \\
& :=\frac{1}{\lambda_{\max }(H)}\left[L(H)-\sum_{i=1}^{s} L_{i}(H)+s \lambda_{\min }(H)\right] .
\end{aligned}
$$

Theorem 3 is an analog of Theorem 2 for this "constant" case. The difference (except for the above-mentioned changes) is that inequality (9) in Theorem 2 is improved to inequality (31), where no number $q \in(0,1)$ is necessary.

Theorem 3. Let $\rho(A)<1, C$ be a fixed positive definite matrix, let matrix $H$ solve the corresponding Lyapunov matrix equation (5), and let

$$
L(H)>0 \text {, }
$$

$$
L(H)-\sum_{i=1}^{s} L_{i}(H)<\lambda_{\max }(H)-s \lambda_{\min }(H) .
$$

Then, $0<\Theta(H)<1$, the system with delay (1) is exponentially stable, and, for an arbitrary solution $x=x(k)$, the estimate

$$
|x(k)| \leq \sqrt{\varphi(H)}\|x(0)\|_{m} \Theta^{\frac{k}{2(m+1)}}(H), \quad k \geq 0,
$$

holds.

Proof. The proof can be done along the lines of the proof of Theorem 2. The inequality $0<\Theta(H)<1$ can be proved in much the same way as the inequality $0<\Theta(k, H) \leq q, k=$ $0,1, \ldots$ Then, the proof can be repeated with $q:=\Theta(H)$.

\section{A Discussion of the Results Obtained, Comparisons, and Examples}

Theorems 2 and 3 provide sufficient conditions for exponential stability. The novelty of our approach is based on a new method of estimating the full difference of Lyapunov function via auxiliary functions $L(k, H), L_{i}(k, H), i=1, \ldots, s$, or via auxiliary constants $L(H), L_{i}(H), i=1, \ldots, s$. Theorem 3 generalizes Theorem 1 in [4].

Some results can detect asymptotic stability but do not provide estimates of solutions, often necessary for computational purposes (in spite of the fact that, for special classes of equations, they can provide criteria on asymptotic stability, depending on delay, of the type "if on only if"). We refer, for example, to the papers $[3,6,14]$. Thus, an advantage over these results is the explicitly expressed estimation of the norm of an arbitrary solution. 
The results are also independent of those in other sources mentioned in the list of references. For example, some new criteria for exponential stability of nonlinear difference systems with time-varying delay were recently proved in [9] where nonlinearities are estimated by linear terms whose matrices are nonnegative and their sum can, for example, be estimated by a constant nonnegative matrix with a spectrum less than 1. For more details, we refer to [9, Theorem 2.2]. Examples 5 and 6 consider linear systems. Unfortunately, an attempt to estimate the right-hand sides of the systems by a nonnegative matrix does not provide a matrix with a spectrum less that 1 and, moreover, none of the cases described by the theorem is applicable. The results in [11] can be considered similarly in the case of absence of stochastic terms.

The exponential stability of linear systems is analyzed in [15] as well, where $\operatorname{det} A \neq 0$ is assumed. In Examples 5 and 6, $\operatorname{det} A=0$. So, the results of [15] are not applicable either.

Discussing how realistic conditions of Theorems 2 and 3 are, we can conclude that, assuming that the norms of matrices $B_{i}$ are small enough, condition (30) of Theorem 3 will be valid if

$$
\lambda_{\max }(H)>\lambda_{\min }(C) .
$$

Condition (31) is equivalent with

$$
\begin{aligned}
(1 & +\varphi(H)) \sum_{j=1}^{s}\left|A^{T} H B_{j}\right|+\varphi(H) \sum_{i, j=1}^{s}\left|B_{i}^{T} H B_{j}\right| \\
& <\lambda_{\text {min }}(C)
\end{aligned}
$$

and is true as well if the above norms are small enough. Thus, crucial for the applicability of Theorem 3 is inequality (33). In such a case, Theorem 3 is applicable in principle. A similar discussion applies with respect to the assumptions of Theorem 2.

In establishing the stability or exponential stability of linear systems with constant coefficients and a single delay, [5] utilizes a different set of sufficient conditions (independent of our results). The main result [5, Theorem 2] has the form of the following theorem.

Theorem 4. Let $\rho(A)<1, C$ be a fixed positive definite symmetric $n \times n$ matrix, let matrix $H$ solve the corresponding Lyapunov matrix equation (5), and, for a fixed $\gamma>1$, let

$$
\begin{aligned}
L_{1}^{*}(H) & >0, \\
L_{2}^{*}(H, \gamma, m) & >0, \\
L_{3}^{*}(H, \gamma, m) & >0,
\end{aligned}
$$

where

$$
\begin{aligned}
L_{1}^{*}(H):= & \lambda_{\max }(H)-\lambda_{\min }(C)+\left|A^{T} H B\right|, \\
L_{2}^{*}(H, \gamma, m):= & \lambda_{\min }(H) \\
& -\gamma^{m+1} \varphi(H)\left[\left|A^{T} H B\right|+\left|B^{T} H B\right|\right],
\end{aligned}
$$

$$
\begin{aligned}
L_{3}^{*}(H, \gamma, m):= & \lambda_{\min }(C)-\left|A^{T} H B\right|-\frac{\gamma-1}{\gamma} \lambda_{\max }(H) \\
& -\gamma^{m} \varphi^{2}(H)\left(\left|A^{T} H B\right|+\left|B^{T} H B\right|\right) .
\end{aligned}
$$

Then, the system with delay

$$
x(k+1)=A x(k)+B x(k-m), \quad k=0,1, \ldots,
$$

is exponentially stable and, for an arbitrary solution $x=x(k)$, the estimate

$$
|x(k)| \leq \sqrt{\varphi(H)}\|x(0)\|_{m} \gamma^{-k / 2}, \quad k \geq 1,
$$

holds.

We compare this result with our Theorem 3 adapted for this case (in its formulation, we set $s=1, m_{1}(k) \equiv m, B_{1} \equiv B$ ). Inequalities $L_{1}^{*}(H)>0$ and $L_{1}(H)>0$ are the same. From inequality (31), we derive

$$
\lambda_{\min }(C)>\left|A^{T} H B\right|+\varphi(H)\left(\left|A^{T} H B\right|+\left|B^{T} H B\right|\right) .
$$

Inequality $L_{3}^{*}(H, \gamma, m)>0$ changes to inequality

$$
\begin{aligned}
\lambda_{\min }(C)> & \left|A^{T} H B\right|+\frac{\gamma-1}{\gamma} \lambda_{\max }(H) \\
& +\gamma^{m} \varphi^{2}(H)\left(\left|A^{T} H B\right|+\left|B^{T} H B\right|\right)
\end{aligned}
$$

and, evidently, is not equivalent with (39). It is more restrictive and can be regarded as equivalent with (39) only if $\varphi(H)=1$ and $\gamma \rightarrow 1^{+}$. In addition, in Theorem 3, an inequality similar to inequality $L_{2}^{*}(H, \gamma, m)>0$ is not necessary.

We demonstrate the results obtained by the following examples.

Example 5. Let $n=2$ and let system (1) be of the form

$$
\begin{aligned}
& x_{1}(k+1)=2 x_{1}(k)+4 x_{2}(k)+b_{2} x_{2}\left(k-m_{2}(k)\right), \\
& x_{2}(k+1)=-x_{1}(k)-2 x_{2}(k)+b_{1} x_{1}\left(k-m_{1}(k)\right),
\end{aligned}
$$

where $k \geq 0$. Using Theorem 3, we prove that the system is exponentially stable if $\left|b_{1}\right|$ and $\left|b_{2}\right|$ are sufficiently small and satisfy

$$
\begin{aligned}
& 27.975991\left|b_{1}\right|+13.988077\left|b_{2}\right|+2498.2520 b_{1}^{2} \\
& +2485.7608\left|b_{1} b_{2}\right|+624.5630 b_{2}^{2}<0.0079925
\end{aligned}
$$

and give an exponential estimate of the norm of solutions. We have

$$
\begin{aligned}
A & =\left(\begin{array}{cc}
2 & 4 \\
-1 & -2
\end{array}\right), \\
B_{1} & =\left(\begin{array}{ll}
0 & 0 \\
b_{1} & 0
\end{array}\right), \\
B_{2} & =\left(\begin{array}{ll}
0 & b_{2} \\
0 & 0
\end{array}\right),
\end{aligned}
$$


and $\rho(A)=0$. Lyapunov equation (5) is satisfied, for example, for

$$
\begin{aligned}
& H=\left(\begin{array}{cc}
1 & 1.99 \\
1.99 & 4
\end{array}\right), \\
& C=\left(\begin{array}{ll}
0.96 & 1.91 \\
1.91 & 3.84
\end{array}\right) .
\end{aligned}
$$

Then, $\lambda_{\text {max }}(H) \doteq 4.9920072, \lambda_{\text {min }}(H) \doteq 0.0079928$, $\lambda_{\text {min }}(C) \doteq 0.0079925$, and $\varphi(H) \doteq 624.5630$. It is easy to find

$$
\begin{aligned}
& \left|A^{T} H B_{1}\right| \doteq 0.0447213\left|b_{1}\right|, \\
& \left|A^{T} H B_{2}\right| \doteq 0.02236078\left|b_{2}\right|, \\
& \left|B_{1}^{T} H B_{1}\right|=4 b_{1}^{2}, \\
& \left|B_{2}^{T} H B_{2}\right|=b_{2}^{2}, \\
& \left|B_{1}^{T} H B_{2}\right|=1.99\left|b_{1} b_{2}\right| .
\end{aligned}
$$

Moreover,

$$
\begin{aligned}
& L(H)=\lambda_{\max }(H)-\lambda_{\min }(C)+\sum_{j=1}^{2}\left|A^{T} H B_{j}\right| \\
& \doteq 4.9920072-0.0079925+0.0447213\left|b_{1}\right| \\
& +0.02236078\left|b_{2}\right| \\
& =4.9840147+0.0447213\left|b_{1}\right|+0.02236078\left|b_{2}\right| \\
& >0 \text {, } \\
& L_{1}(H) \\
& =\lambda_{\min }(H)-\varphi(H)\left[\left|A^{T} H B_{1}\right|+\sum_{j=1}^{2}\left|B_{1}^{T} H B_{j}\right|\right] \\
& \doteq 0.0079928 \\
& -624.5630\left(0.0447213\left|b_{1}\right|+4 b_{1}^{2}+1.99\left|b_{1} b_{2}\right|\right) \\
& \doteq 0.0079928-27.93127\left|b_{1}\right|-2498.2520 b_{1}^{2} \\
& -1242.8804\left|b_{1} b_{2}\right|, \\
& L_{2}(H) \\
& =\lambda_{\min }(H)-\varphi(H)\left[\left|A^{T} H B_{2}\right|+\sum_{j=1}^{2}\left|B_{2}^{T} H B_{j}\right|\right] \\
& \doteq 0.0079928 \\
& -624.5630\left(0.02236\left|b_{2}\right|+1.99\left|b_{1} b_{2}\right|+b_{2}^{2}\right) \\
& =0.0079928-13.965716\left|b_{2}\right|-1242.8804\left|b_{1} b_{2}\right| \\
& -624.5630 b_{2}^{2} \text {. }
\end{aligned}
$$

Now Theorem 3 is applicable. Assumption (30) is fulfilled and assumption (31) is valid as well because

$$
\begin{aligned}
& L(H)-\sum_{i=1}^{2} L_{i}(H) \\
& \doteq 4.9680291+27.975991\left|b_{1}\right|+13.988077\left|b_{2}\right| \\
&+2498.2520 b_{1}^{2}+2485.7608\left|b_{1} b_{2}\right| \\
&+624.5630 b_{2}^{2}, \\
& \lambda_{\max }(H)-2 \lambda_{\min }(H) \doteq 4.9920072-0.0159856 \\
& \doteq 4.9760216
\end{aligned}
$$

and inequality

$$
\begin{aligned}
& 27.975991\left|b_{1}\right|+13.988077\left|b_{2}\right|+2498.2520 b_{1}^{2} \\
& +2485.7608\left|b_{1} b_{2}\right|+624.5630 b_{2}^{2}<0.0079925
\end{aligned}
$$

holds due to (42). In view of the last inequality, we have

$$
\begin{aligned}
& \Theta(H)=\frac{1}{\lambda_{\max }(H)}\left[L(H)-\sum_{i=1}^{2} L_{i}(H)+2 \lambda_{\min }(H)\right] \\
& \quad<\frac{1}{624.5630}[4.968029+0.0079925+0.0159856] \\
& \quad \doteq 0.0079928<1 .
\end{aligned}
$$

For an arbitrary solution $x(k)$, the estimate

$$
\begin{aligned}
&|x(k)| \leq \sqrt{\varphi(H)}\|x(0)\|_{m} \Theta^{k / 2(m+1)}(H) \\
& \doteq 24,99126(0.0079928)^{k / 2(m+1)}\|x(0)\|_{m}, \\
& k \geq 0,
\end{aligned}
$$

holds.

Example 6. Let $n=2$ and let system (1) be of the form

$$
\begin{aligned}
x_{1}(k+1)= & 2 x_{1}(k)+4 x_{2}(k) \\
& +0.0005 \cos \left(\frac{1}{k+1}\right) x_{2}\left(k-m_{2}(k)\right), \\
x_{2}(k+1)= & -x_{1}(k)-2 x_{2}(k),
\end{aligned}
$$

where $k \geq 0$. Using Theorem 2 , we prove that the system is exponentially stable and, for an arbitrary solution $x(k)$, the estimate

$$
\begin{array}{r}
|x(k)| \leq 24,99126(0.99984)^{\frac{k}{2(m+1)}}\|x(0)\|_{m}, \\
\quad k \geq 0,
\end{array}
$$


holds. Because the system differs from the system considered in Example 5 only in the matrices of the delayed terms where

$$
\begin{aligned}
& B_{1}(k)=\left(\begin{array}{ll}
0 & 0 \\
0 & 0
\end{array}\right), \\
& B_{2}(k)=\left(\begin{array}{cc}
0 & 0.0005 \cos \left(\frac{1}{k+1}\right) \\
0 & 0
\end{array}\right),
\end{aligned}
$$

it is possible to use some previous computations with the same matrices $C$ and $H$. We obtain

$$
\begin{aligned}
& \left|A^{T} H B_{2}(k)\right| \doteq 0.00001118 \cos \left(\frac{1}{k+1}\right), \\
& \left|B_{2}^{T}(k) H B_{2}(k)\right| \doteq 0.00000025 \cos \left(\frac{1}{k+1}\right) \text {, } \\
& L(k, H)=\lambda_{\max }(H)-\lambda_{\min }(C)+\left|A^{T} H B_{2}(k)\right| \\
& \doteq 4.9840147+0.00001118 \cos \left(\frac{1}{k+1}\right), \\
& L_{1}(k, H)=\lambda_{\min }(H)=0.0079928, \\
& L_{2}(k, H) \\
& =\lambda_{\min }(H) \\
& -\varphi(H)\left[\left|A^{T} H B_{2}(k)\right|+\left|B_{2}^{T}(k) H B_{2}(k)\right|\right] \\
& \doteq 0.0079928-0.0071390 \cos \left(\frac{1}{k+1}\right) \text {. }
\end{aligned}
$$

Inequality (8) obviously holds. Inequality (9) takes the form

$$
\begin{array}{r}
4.9840147+0.0071502 \cos \left(\frac{1}{k+1}\right) \leq 4.9920072 q, \\
k \geq 0,
\end{array}
$$

and is valid, for example, for $q=0.99984$. From (10), we derive inequality (52).

Remark 7. As noted above, the results of the recent paper [9] are not applicable to Example 5 or 6 . We will explain this using Example 6. To apply, for example, Theorem 2.3 in [9] we develop the best possible estimations of the right-hand sides of (51) (below, the notation of the above-mentioned paper is used). We get

$$
\begin{aligned}
& \mid 2 x_{1}(k)+4 x_{2}(k) \\
& \quad+0.0005 \cos \left(\frac{1}{k+1}\right) x_{2}\left(k-m_{2}(k)\right)|\leq 2| x_{1}(k) \mid \\
& \quad+4\left|x_{2}(k)\right|+0.0005\left|x_{2}\left(k-m_{2}(k)\right)\right| \\
& \left|-x_{1}(k)-2 x_{2}(k)\right| \leq\left|x_{1}(k)\right|+2\left|x_{2}(k)\right|
\end{aligned}
$$

Then,

$$
\begin{aligned}
& A_{0}=\left(\begin{array}{ll}
2 & 4 \\
1 & 2
\end{array}\right), \\
& A_{1}=\left(\begin{array}{cc}
0 & 0.0005 \\
0 & 0
\end{array}\right) .
\end{aligned}
$$

The eigenvalues of the matrix $A_{0}+A_{1}$ are $\lambda_{1} \approx-0.00012$, $\lambda_{2} \approx 4.00012$. The inequality $\rho\left(A_{0}+A_{1}\right)<1$, necessary for exponential stability, does not hold and Theorem 2.3 is not applicable. Similarly, we can show that neither Theorem 2.2 nor Theorem 2.4 is applicable and, therefore, such results are independent of the presented results. Similarly, the results of the papers $[1,2,7,8,10]$ are not applicable either since, in an analysis in [9], the authors show that their results are more general. Then, it is obvious that our results are independent of these results as well.

\section{Conflicts of Interest}

The author declares that he has no conflicts of interest.

\section{Acknowledgments}

The author was supported by the Czech Science Foundation under the Project 16-08549S. This work was realized in Central European Institute of Technology (CEITEC) with research infrastructure supported by a project financed from European Regional Development Fund (Project CZ.1.05/1.1.00/02.0068).

\section{References}

[1] R. P. Agarwal, Y.-H. Kim, and S. K. Sen, "New discrete Halanay inequalities: stability of difference equations," Communications in Applied Analysis. An International Journal for Theory and Applications, vol. 12, no. 1, pp. 83-90, 2008.

[2] M. Buslowicz, "Simple stability conditions for linear positive discrete-time systems with delays," Bulletin of the Polish Academy of Sciences Technical Sciences, vol. 56, pp. 325-328, 2008.

[3] J. Čermák, "Stability conditions for linear delay difference equations: a survey and perspective," Tatra Mountains Mathematical Publications, vol. 63, pp. 1-29, 2015.

[4] J. Diblík, D. Y. Khusainov, J. Baštinec, and A. S. Sirenko, "Exponential stability of linear discrete systems with constant coefficients and single delay," Applied Mathematics Letters, vol. 51, pp. 68-73, 2016.

[5] J. Diblík, D. Y. Khusainov, J. Baštinec, and A. S. Sirenko, "Stability and exponential stability of linear discrete systems with constant coefficients and single delay," Applied Mathematics and Computation, vol. 269, Article ID 21439, pp. 9-16, 2015.

[6] M. M. Kipnis and D. A. Komissarova, "A note on explicit stability conditions for autonomous higher order difference equations," Journal of Difference Equations and Applications, vol. 13, no. 5, pp. 457-461, 2007.

[7] E. Liz, "Stability of non-autonomous difference equations: simple ideas leading to useful results," Journal of Difference Equations and Applications, vol. 17, no. 2, pp. 203-220, 2011. 
[8] E. Liz and J. B. Ferreiro, "A note on the global stability of generalized difference equations," Applied Mathematics Letters, vol. 15, no. 6, pp. 655-659, 2002.

[9] P. H. Ngoc and L. T. Hieu, "New criteria for exponential stability of nonlinear difference systems with time-varying delay," International Journal of Control, vol. 86, no. 9, pp. 16461651, 2013.

[10] S. Udpin and P. Niamsup, "New discrete type inequalities and global stability of nonlinear difference equations," Applied Mathematics Letters, vol. 22, no. 6, pp. 856-859, 2009.

[11] L. Xu and S. S. Ge, "Exponential ultimate boundedness of nonlinear stochastic difference systems with time-varying delays," International Journal of Control, vol. 88, no. 5, pp. 983-989, 2015.

[12] S. N. Elaydi, "An introduction to difference equations," in Undergraduate Texts in Mathematics, Springer, 3rd Edition edition, 2005.

[13] R. P. Agarwal, Difference Equations and Inequalities: Theory, Methods, and Applications, vol. 228, CRC Press, 2000.

[14] J. Čermák and P. Tomášek, "On delay-dependent stability conditions for a three-term linear difference equation," Funkcialaj Ekvacioj. Serio Internacia, vol. 57, no. 1, pp. 91-106, 2014.

[15] A. S. Bychkov and D. Y. Khusainov, "Exponential convergence estimates for delay difference systems," Journal of Differential Equations, vol. 38, no. 9, pp. 1368-1370, 2002. 


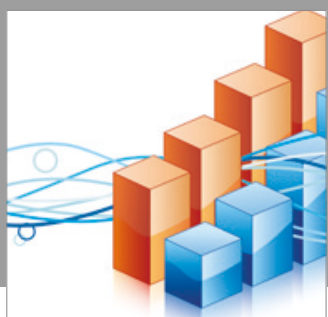

Advances in

Operations Research

vatersals

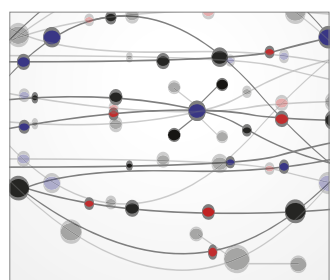

\section{The Scientific} World Journal
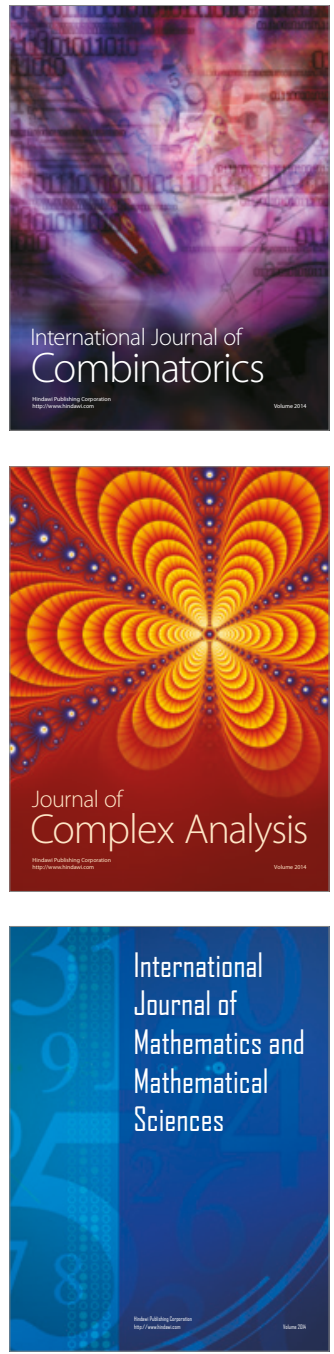
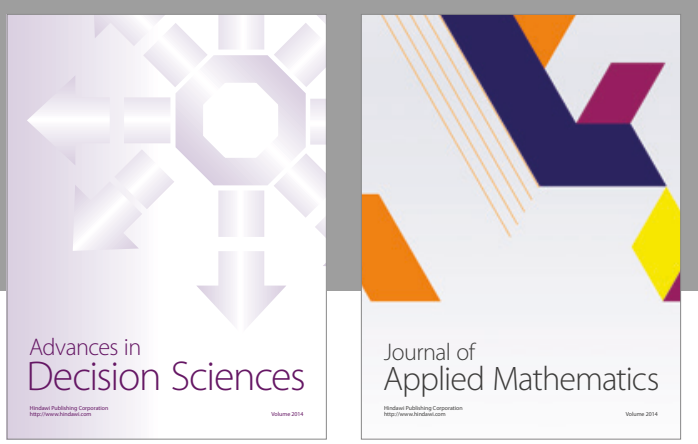

Algebra

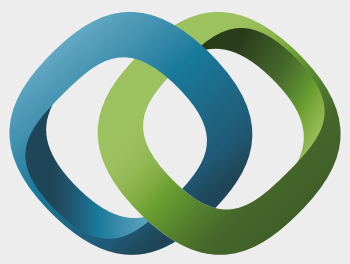

\section{Hindawi}

Submit your manuscripts at

https://www.hindawi.com
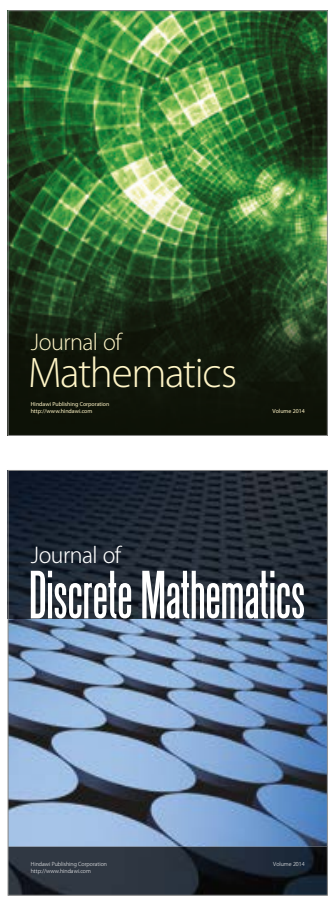

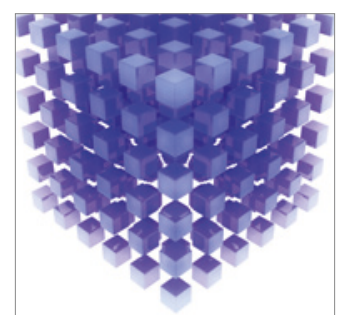

Mathematical Problems in Engineering
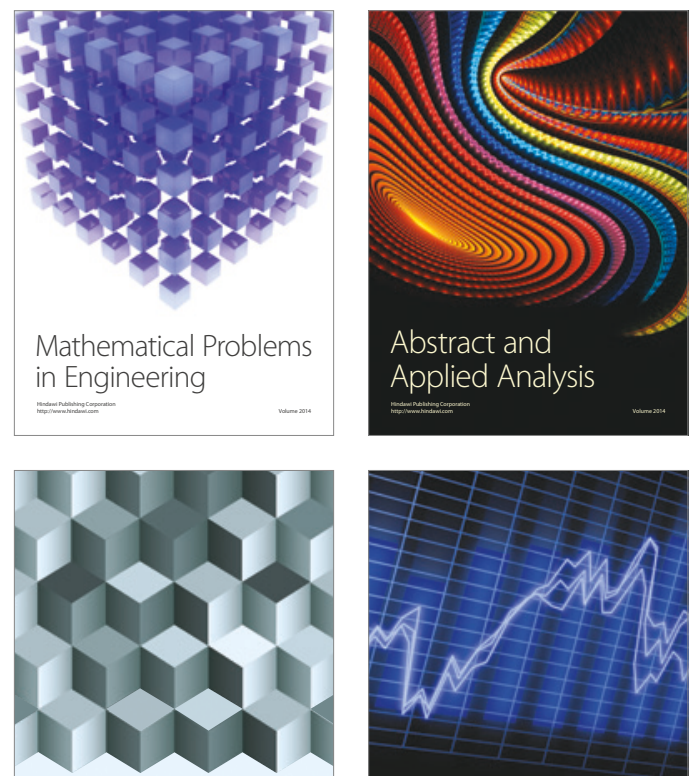

Journal of

Function Spaces

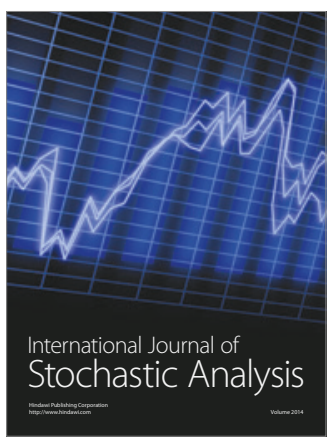

Probability and Statistics
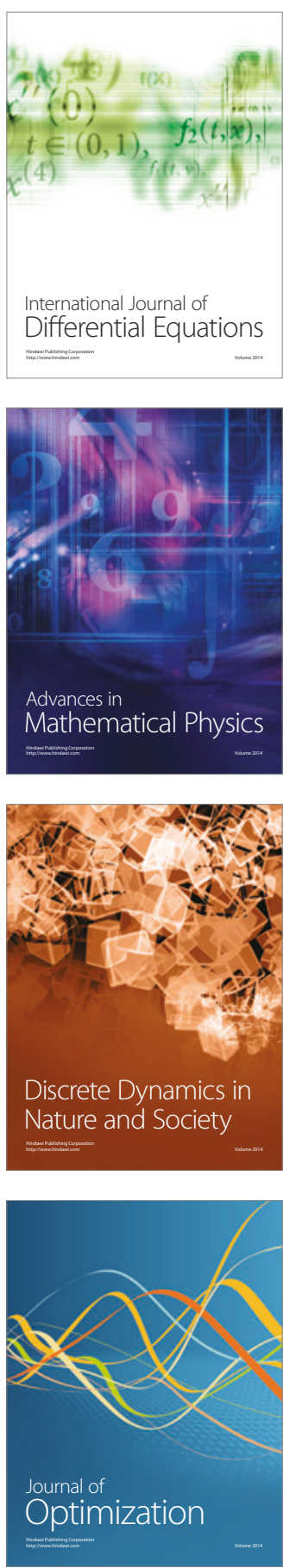